\title{
Mulberry Leaf Extract Alleviates Staphylococcus Aureus-Induced Conjunctivitis in Rabbits via Downregulation of NLRP3 Inflammaome and Upregulation of the Nrf2 System and Suppression of Pro-Inflammatory Cytokines
}

\section{Chen Ying}

Zunyi Medical University - Zhuhai Campus

\section{Linglin Lai}

Zunyi Medical University - Zhuhai Campus

\section{Zhentao Mo}

Zunyi Medical University - Zhuhai Campus

\section{Ennian Leng}

Zunyi Medical University - Zhuhai Campus

Yueyue Zhang

Zunyi Medical University - Zhuhai Campus

Xu Li

Zunyi Medical University - Zhuhai Campus

Yiqi Li

Zunyi Medical University - Zhuhai Campus

Wenna Li ( $\square$ 26153767@qq.com )

Zunyi Medical University - Zhuhai Campus

\section{Research}

Keywords: Mulberry leaf extract, Staphylococcus aureus, Bacterial conjunctivitis, NOD-like receptor leucine-rich pyrin domain-containing protein 3 inflammasome, nuclear factor erythroid 2-related factor 2 , pro-inflammatory cytokines

Posted Date: July 13th, 2021

DOI: https://doi.org/10.21203/rs.3.rs-665199/v1

License: (9) This work is licensed under a Creative Commons Attribution 4.0 International License. Read Full License 


\section{Abstract}

Mulberry leaves are widely used in traditional Chinese medicine for their antioxidant, anti-inflammatory, antibacterial, antiobesity, antidiabetic, antiatherosclerotic, and anticancer properties. The current study aimed to investigate the effect of mulberry leaf extract (MLE) on Staphylococcus aureus (S. aureus)induced conjunctivitis ( $5 \times 10^{9} \mathrm{CFU}, 0.5 \mathrm{~mL} /$ eye) in a rabbit model. Rabbits were treated with MLE (5 $\mathrm{mL} / \mathrm{kg} \cdot \mathrm{d}^{-1}$ and $10 \mathrm{~mL} / \mathrm{kg} \cdot \mathrm{d}^{-1}$ ), $0.9 \%$ saline, or pearl bright eye drops (PBE) for 5 days. The results showed that MLE treatment significantly reduced the clinical sign scores of conjunctivitis, alleviated clinical signs, and decreased bacterial load and histological damage in a time- and dose-dependent manner compared to that in the conjunctivitis control group. The antibacterial and anti-inflammatory activities of MLE (10 $\mathrm{mL} / \mathrm{kg} \cdot \mathrm{d}^{-1}$ ) were equal to or greater than those of the positive control drug PBE. In addition, MLE significantly decreased the levels of pro-inflammatory cytokines, downregulated the NOD-like receptor leucine-rich pyrin domain-containing protein 3 (NLRP3) inflammasome, and upregulated the nuclear factor erythroid 2-related factor 2 (Nrf2) system. Overall, MLE is effective in alleviating $S$. aureus-induced conjunctivitis in rabbits and this mechanism is associated with the inhibition of the NLRP3 inflammasome and activation of the Nrf2 system to regulate pro-inflammatory signaling.

\section{Introduction}

Conjunctivitis is a common medical condition characterized by red eyes, congestion, exudation, and edema. Specifically, bacterial conjunctivitis is one of the most diagnosed eye diseases by general practitioners and is characterized by mucopurulent discharge and conjunctival hyperemia.

Staphylococcus aureus (S. aureus) is one of the most common pathogens to cause bacterial conjunctivitis and is capable of infecting the eyelid, conjunctiva, and cornea [1-3]. The conventional treatments for bacterial conjunctivitis include anti-bacterial- and/or immunosuppressive/steroid eyedrops. However, some studies have reported resistance to these treatments $[4,5]$. Therefore, there is an urgent need for more reliable and effective drugs.

Episodes of infection with acute bacterial conjunctivitis can lead to inflammation [6, 7]. Thus, the effect of MLE on conjunctivitis may involve the suppression of inflammatory mediators in rabbits. The NOD-like receptor leucine-rich pyrin domain-containing protein 3 (NLRP3) inflammasome, as the first line of defense against external threats, is activated when it identifies certain stress factors, such as pathogens (Artlett, 2013). The oxidative insult contributes to the development of responses of allergic conjunctivitis [8]. Meanwhile, the nuclear factor erythroid 2-related factor 2 (Nrf2) pathway, as the major regulator of oxidative stress, is also believed to play an important role in the pathogenesis of common inflammation [9]. Previous study has provided that oxidative stress and inflammation coexisted in the conjunctiva of patients with Atopic Keratoconjunctivitis [10]. Multiple lines of evidence suggest that there is a crosstalk between the Nrf2 and inflammasome pathways at different levels, which are associated with many inflammatory processes, including acute and chronic inflammation, reactive oxygen species, and autophagy [11-13]. Yet, the contribution of NLRP3 inflammasome and Nrf2 pathway to bacterial ocular infection remains unclear. 
Mulberry leaves consist of the dried leaves of Morus alba L., a plant belonging to the Moraceae family. They are approved by the Ministry of Health of the People's Republic of China as medicine and food homology. According to traditional Chinese medicine records, mulberry leaves are prescribed with chrysanthemum, Scutellaria, and cassia seeds to purge toxins from the liver, improve vision, and treat eye diseases [14]. A few experimental studies have also reported the effectiveness of mulberry leaves in treating eye conditions resulting from diabetes mellitus [15-17]. A previous study found that mulberry leaf extract (MLE) could ameliorate the pathological alterations of retinal neurons and neurotransmitters of cubs of either diabetic and/or hypercholesterolemic rats [15]. Furthermore, polyphenol-rich MLE could attenuate early retinopathy in diabetic maternal rats and cubs through its antioxidant, anti-inflammatory, anti-apoptotic, and anti-angiogenic effects [17]. Another study [16] also showed that aqueous MLE could protect diabetic rats against the degeneration of retinal neuronal cells, which might be attributed to its potential antioxidant activity. However, to date, little is known about the effects and mechanism of MLE on bacterial conjunctivitis.

Pearl bright eye drops (PBE) is an over-the-count eye drop in Chinese patent medicine [14]. Pearl hydrolysate and borneol are the main components of PBE formulation, and its pharmaceutical excipients including: sodium chloride, ethylparaben, alcohol, and sodium hyaluronate et al. [18, 19]. The PBE properties of calming the nerves and tranquilizing the mind [20], clearing heat, improving eyesight, and relieving pain $[21,22]$ have a certain therapeutic potential for bacterial or allergic conjunctivitis, dry eye disease, and asthenopia [14].

Based on previous evidence, we hypothesized that as mulberry leaves have anti-inflammatory, antioxidant, and antibacterial activities and have been successfully used in eye diseases, they may protect against conjunctivitis. Therefore, to study the efficacy of mulberry leaves in the treatment of bacterial conjunctivitis, we used mulberry leaf aqueous extract to treat rabbits with conjunctivitis caused by $S$. aureus with PBE as a positive control. Meanwhile, to further explore the underlying mechanisms, we characterized the roles of NLRP3 inflammasome and Nrf2 pathway in the protection of MLE and PBE to bacterial conjunctivitis.

\section{Material And Methods \\ 2.1. Chemicals}

Mulberry leaves (batch no. 06060101) were purchased from Hubei Jingui Industrial Development Co., Ltd. (Hubei, China). MLE and MLE eye drops were prepared as follows: roasted mulberry leaves $(200 \mathrm{~g})$ were decocted in $1 \mathrm{~L}$ of sterile water at $100^{\circ} \mathrm{C}$ for $2 \mathrm{~h}$. Next, the water extract was cooled and filtered through Whatman No. 1 filter paper to generate MLE. The pH of MLE was adjusted to 7.0 using boric acid buffer $(12.4 \mathrm{~g} / \mathrm{L})$ and borax buffer $(19.1 \mathrm{~g} / \mathrm{L})$. Sodium chloride was added to the resulting solution $(0.9 \% \mathrm{wt} / \mathrm{vol})$ and the solution was filtered using a $0.22 \mu \mathrm{m}$ membrane to produce MLE eye drops $(0.1 \mathrm{~L})$. All the solutions mentioned above were stored at $4^{\circ} \mathrm{C}$ before the experiment. Pearl bright eye drops (its main components are pearl hydrolysate and borneol, PBE, batch no. 20190726) were obtained and produced 
by Suzhou Industrial Park Tianlong Pharmaceutical Co., Ltd. (Suzhou, China). S. aureus ([CMCC(B)26003]) was obtained from the Research Center for Biological of Zhuhai Campus, Zunyi Medical University. S. aureus solution was incubated at $37^{\circ} \mathrm{C}$ for $18 \mathrm{~h}$, centrifuged at $3000 \mathrm{r} / \mathrm{min}$ for 10 min, and diluted to a concentration of $5 \times 10^{9}$ colony-forming units (CFU) $/ \mathrm{mL}$ with normal saline in sterile tubes.

\subsection{Animal experiments}

New Zealand rabbits of both sexes $(2.50 \sim 3.00 \mathrm{~kg})$ were obtained from the Animal Laboratory Center of Zhuhai Campus, Zunyi Medical University. Animals were housed one per cage, provided a laboratory diet (Animal Center of Zhuhai campus, Zunyi Medical University, Zhuhai, China) and water ad libitum. All experiments procedures and animal welfare were carried out in accordance with the "Guidelines for the Management and Use of Laboratory Animals"(Ministry of Science and Technology of China, 2006) and approved by the Zhuhai Campus Zunyi Medical University Institutional Animal Ethics and Utilization Committee. After one week of adaptation in laboratory conditions $\left(24 \pm 2^{\circ} \mathrm{C}, 60 \pm 5 \%\right.$ relative humidity, and 12-h light/dark cycle), healthy rabbits (without eye disease) were assigned randomly to 5 groups (with 5 animals in each group and utilized both eyes of each animal) as follows: control group (normal saline), model group (normal saline), low-dose MLE group ( $\left.5 \mathrm{~mL} / \mathrm{kg} \cdot \mathrm{d}^{-1}, \mathrm{LMLE}\right)$, high-dose MLE group (10 $\mathrm{mL} / \mathrm{kg} \cdot \mathrm{d}^{-1}, \mathrm{HMLE}$ ), and positive control group (pearl bright eye drops, PBE). $S$. aureus conjunctivitis was induced according to previously described methods [23-25] with a slight modification. Briefly, the conjunctivas of each white rabbit (excluding the control group) were scratched with a No. 9 sterile needle and $S$. aureus $(0.5 \mathrm{~mL} /$ eye) was topically injected onto the conjunctiva and retained for $30 \mathrm{~s}$ to ensure the uniform spearing of the bacteria (Day 0 ). Treatment was initiated on the second day after $S$. aureus inoculation (Day 1). The MLE eye drops, PBE, or sterile normal saline was applied dropwise onto the superior palpebral and bulbar conjunctiva of the right and left eyes of rabbits $(0.1 \mathrm{~mL} /$ eye three times daily), and LMLE, HMLE or normal saline $\left(10 \mathrm{~mL} / \mathrm{kg}^{\prime} \mathrm{d}^{-1}\right)$ was administered by oral gavage once a day for 5 days respectively. The eyes of rabbits in the "positive control group" were given PBE, after the same volume of normal saline was orally administered as above-metioned.

\subsection{Animal Scarifice}

At the end of the experiment, rabbits were euthanized by intravenous injection with excessive sodium pentobarbital. The palpebral conjunctiva of right eyes of three rabbits in each group were randomly sclected and taken by sterile scalpel for histopathologic analysis. The remaining samples were removed immediately after euthanasia, frozen by liguid nitrogen and stroed at $-80^{\circ} \mathrm{C}$ for further analysis.

\subsection{Scoring criteria of the pathological changes in the conjunctiva}

A double-blind trial was performed to evaluate the pathological changes in the conjunctiva. Congestival redness, discharge and lid edema were assessed daily by three independent blinded investigators using a $0-3$ scale according to previously described criteria $[26,27]$, in which a 0 score indicates no signs; 1 , mild 


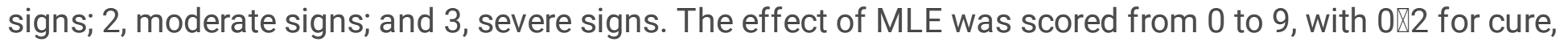
2\5 for significant effect, 5囚8 for improvement, $8 \sim 9$ for ineffectiveness.

\subsection{Bacterial examination of the eyelid conjunctiva}

To assess bacterial growth inhibition, samples were collected from both the right and left eyes of rabbits with a sterile cotton swab without the administration of any topical anesthetic agents on Days 0,1 , and 5 . The corneal and mucosal surfaces of the conjunctival fornix were swabbed without touching the preocular skin or hair. After sampling, blood agar medium plates were immediately coated with each sample and incubated at $37^{\circ} \mathrm{C}$ for $28 \mathrm{~h}$ to obtain bacterial cultures at mid-log growth phase. After incubation, the colony formation of bacteria in every sample was monitored by counting number of bacterial colonies formed.

\subsection{Histological examination of the conjunctiva}

The conjunctiva tissues were fixed with $4 \%$ paraformaldehyde for $18 \mathrm{~h}$, followed by embedding in paraffin. Consecutive 4- $\mu \mathrm{m}$ paraffin sections were cut and stained with hematoxylin-eosin to visualize tissue morphology and cellular infiltrates.

\subsection{Quantitative real-time reverse transcription PCR (qPCR)}

The expression of the following genes in the conjunctiva was studied using qPCR: Caspase-1 (CASP-1), ASC, NLRP3, Interleukin-18 (IL-18), Interleukin-6 (IL-6), and Interleukin-1 beta (IL-1 $\beta$ ). Primer pairs (Sangon, Shanghai, China) are listed in Table 1. Taken isolated palpebral conjunctiva, and RNA isolation (Total

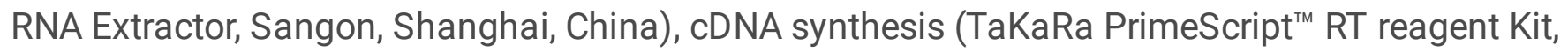
Baosheng, Dalian, China), and amplification (TaKaRa Ex Taq PCR Kit, Baosheng, Dalian, China) were performed according to the manufacturer's instructions. The reaction mixture was preheated for $1 \mathrm{~min}$ at $95^{\circ} \mathrm{C}$ to activate the Taq DNA polymerase, followed by 45 cycles of $3 \mathrm{~s}$ at $95^{\circ} \mathrm{C}$ and $34 \mathrm{~s}$ at $60^{\circ} \mathrm{C}$. Throughout the qPCR analysis, product identities were confirmed using a $60-95^{\circ} \mathrm{C}$ melting curve analysis. All samples were run in duplicate using an Mx3005P Real-Time PCR system (Agilent, Santa Clara, CA, USA). The changes in mRNA expression for the internal standard (GAPDH) were calculated according to the $2^{-\triangle \triangle C T}$ method (CT, cycle threshold).

\subsection{Western blot}

Conjunctival protein was extracted using a Protease Inhibitor Cocktail kit (Beyotime, China) and quantified using the BCA Protein Assay kit (Beyotime). The equivalent proteins of each group were separated using polyacrylamide gel electrophoresis and transferred to PVDF membranes (Bio-Rad, Hercules, CA, USA). The membranes were blocked with $5 \%$ nonfat milk and then incubated with the primary antibody (1:1000, CST, Danvers, MA, USA) and secondary antibodies conjugated to horseradish peroxidase (1:2500, Abcam, Cambridge, UK) at $4^{\circ} \mathrm{C}$ overnight. Band detection was thereafter revealed by enhanced chemiluminescence using electrochemiluminescence western blot detection reagents. Protein quantification was performed using densitometry and Quantity One software. $\beta$-actin was used as an internal control. 


\subsection{Statistical analysis}

The statistical software SPSS v.18.0 (IBM SPSS, Inc., Chicago, IL, http://www.ibm.com) was used to analyze the data and experimental results are presented as mean \pm SEM. Statistical differences were analyzed using independent sample t-tests, and the differences were considered to be significant when $\mathrm{P}$ $\leq 0.05$.

\section{Results}

\subsection{Clinical signs of bacterial conjunctivitis in rabbits}

Rabbit eyes were observed before and after $S$. aureus infection to determine whether the conjunctival infection model had been successfully established. Chemosis, exudate, eyelid gluing, diffuse conjunctival hyperemia, and eyelid edema were generally increased in infected rabbits after Day 1 (Fig. 1 b-e); and there were no significant differences among the infected groups. The major inflammatory signs in $S$. aureus-infected eyes were tearing, redness, lid edema, chemosis, mucopurulent discharge, and iris erythema. The results showed that the model of acute infectious conjunctivitis was successfully established with obvious inflammatory reactions. To assess the effect of the drugs, rabbit eyes treated with LMLE, HMLE, and PBE were reevaluated on Day 5 (Fig. 1h, i, j), and clinical signs of conjunctivitis were apparently alleviated in all treated groups compared to the model group. HMLE decreased congestion, secretion, and edema in levels comparable to the positive control drug PBE. These results indicate that MLE had a good therapeutic effect on bacterial conjunctivitis in rabbits, especially at high doses.

\subsection{Effect of MLE on conjunctivitis clinical sign scores in rabbits}

In order to evaluate the therapeutic effect of MLE, the pathological inflammatory changes in the conjunctiva were evaluated according to the scoring criteria (Table 2), with PBE used as a positive control. The clinical sign scores were concordant with the clinical signs (Fig. 2) and were significantly increased on Day 0 in all infected groups compared to the control group. However, in the three treated groups, the increase was significantly reduced on Day 2 compared to the model group $(P<0.01)$. On Day 5 , the inflammatory signs (congestion, secretion, and edema) persisted in the model group but had generally disappeared in the HMLE and PBE groups $(P<0.01)$. The results showed that HMLE exhibited equal anti-inflammatory activity than that of the positive control drug PBE in a time-dependent manner ( $P$ $<0.01)$.

\subsection{Effect of MLE on bacterial growth inhibition in the conjunctiva}


Bacterial colony formation assay was performed to determine the anti-bacterial effect of MLE and PBE. All infected groups showed lawn of bacterial growth on Day 0, while the control group did not (Fig. 3A). On Day 5 , while the model group still showed considerable bacterial growth, significant inhibition of colony formation was observed in the three treated groups. The HMLE and PBE group cultures had an appearance comparable to that of the control group and showed almost $100 \%$ inhibition of colony formation and bacterial growth, while the LMLE group showed $80 \%$ inhibition (Fig. 3B). The bacterial negative conversion rate in eyes of each rabbit was also counted on Days 0,1 , and 5 by observing number of bacterial colonies formed. As shown in Fig. 3C, on Day 1, it was $10 \%, 20 \%$, and $20 \%$ in the LMLE, HMLE, and PBE groups, respectively; however, on Day 5 , it was $70 \%, 90 \%$, and $90 \%$, respectively. These results revealed that MLE appeared to contain components with significant bacterial growthinhibition effect.

\subsection{Effect of MLE on histological features of infectious conjunctivitis}

The attenuating effects of MLE on the clinical scores of bacterial conjunctivitis led us to further investigate the histology of the conjunctiva. Hematoxylin-eosin staining (Fig. 4) demonstrated that severe telangiectasia, capillary congestion, edema, and lymphocyte infiltration were present in model group. After 5 days of treatment, inflammation was alleviated to different degrees in all treated groups and the prominent inhibitory effects of HMLE and PBE were comparable to that of the model group. These findings suggested that MLE was efficacious in the treatment of $S$. aureus-induced conjunctival inflammation in a dose-dependent manner.

\subsection{Effect of MLE on NLRP3 inflammasome and inflammation-related gene expression in the conjunctiva}

Owing to the central regulating role of the NLRP3 inflammasome in the pathogenesis of inflammation, the ability of each of the drugs to reduce its gene expression levels (Caspase-1 (Fig. 5a), ASC (Fig. 5b), and NLRP3 (Fig. 5c)) was analyzed using qPCR in the conjunctivitis rabbit model on Day 5 , as was their ability to decrease the mRNA expression levels of downstream pro-inflammatory cytokines, IL-18 (Fig. 5d), IL-6 (Fig. 5e), and IL-1 $\beta$ (Fig. 5f). As displayed in Fig. 5, the NLRP3 inflammasome and its downstream pro-inflammatory cytokine mRNA expression levels were significantly enhanced in the model group when compared to those obtained in the control group $(P<0.05)$. Figure 5 also shows that the gene expression levels of NLRP3 inflammasome and pro-inflammatory cytokines had returned to normal on Day 5 in the groups of animals treated with HMLE and PBE. These results showed that the NLRP3 inflammasome and its downstream pro-inflammatory cytokine mRNA levels expressed by MLE-treated conjunctiva in bacterial conjunctivitis rabbit models significantly decreased in a dose-dependent manner.

\subsection{Effect of MLE on the activation of NLRP3 inflammasome and downstream inflammatory factors in}




\section{conjunctivitis}

In view of the suppressive effects of the drugs on the NLRP3 inflammasome and inflammation-related gene expression, their effects on protein synthesis of the NLRP3 inflammasome and inflammatory factors in the conjunctiva were evaluated after 5 days of treatment using western blotting (Fig. 6a-b) and statistical analysis of the blots (Fig. $6 \mathrm{c}-\mathrm{i})$. The results obtained were in accordance with the foregoing observations, where the expression levels of NLRP3 inflammasome proteins [Caspase-1 (Fig. 6C), NLRP3 (Fig. 6d), and ASC (Fig. 6e)] and inflammatory-related proteins [TNF-a (Fig. 6f), IL-1ß (Fig. 6g), IL-6 (Fig. 6h), and IL-18 (Fig. 6i)] were significantly increased in the model group compared to those in the control group but was reversed in the three treated groups. In addition, HMLE had stronger effects on NLRP3 inflammasome and inflammatory-related protein synthesis than LMLE, which were not significantly different $(P>0.05)$ from those of the positive control drug, PBE.

\subsection{Effect of MLE on the activation of Nrf2 in conjunctivitis}

In our study, western blotting showed that MLE treatment ( 5 and $10 \mathrm{~mL} / \mathrm{kg} \cdot \mathrm{d}^{-1}$ ) downregulated the expression of Keap1 (Fig. 7b) and upregulated the expression of Nrf2 (Fig. 7c) in conjunctiva tissue (P< $0.05)$, suggesting that MLE treatment could significantly activate the Nrf2 signaling pathway. At the same time, PBE administration, used as a positive control, significantly elevated Nrf2 expression $(P<0.05)$ but did not affect Keap1 expression $(P>0.05)$.

\section{Discussion}

Mulberry leaves have been used for thousands of years in traditional Chinese medicine for several conditions, including diabetes, hyperlipidemia, atherosclerosis, obesity, and inflammation as well as urinary and nervous system diseases [28-32]. Approximately 100 bioactive phytochemicals have been isolated from mulberry leaves, including organic acids, phenolic acids, flavonoids, chalcones, coumarins, polyhydroxyalkaloids, catechins, terpenoids, and stilbenoids [33]. Results form clinical and experimental studies have clearly revealed that extracts or compounds of mulberry leaves possess significant antiinflammatory, antioxidant, and antibacterial effects [33,34]. The effectiveness of mulberry leaves for major eye diseases has been documented according to experimental data and records of traditional Chinese medicine [14-17]. Nonetheless, evidence demonstrating the beneficial effects of mulberry leaves against bacterial conjunctivitis and the related molecular mechanisms remain scarce. This is the first study to demonstrate that MLE effectively inhibited the NRLP3 inflammasome pathway and activated the Nrf2 pathway, leading to reduced conjunctiva inflammation in a rabbit model of bacterial conjunctivitis induced by $S$. aureus.

S. aureus-induced conjunctivitis in a rabbit model exhibited clinical signs similar to those of acute bacterial conjunctivitis in humans, such as lid edema, conjunctival erythema, tearing, chemosis, and mucopurulent discharge [35]. Treatment with MLE noticeably reduced clinical sign scores, alleviated clinical signs, and decreased bacterial colonies formation and histological damage in a time- and dose- 
dependent manner, indicating its protective effect against bacterial conjunctivitis. Based on the theory of traditional Chinese medicine, conjunctivitis is mainly caused by toxic-heat and damp-heat accumulated in the eyes during the first or acute attack. Therefore, as mulberry leaves have detumescence, pain-relieving, liver-clearing, and vision-improving activities [14], they have significant therapeutic effects in acute conjunctivitis.

As we stated above, our results also showed that the positive control drug, PBE, exhibited equal healing effect against bacterial conjunctivitis than that of HMLE. A recent study has proven that the major antimicrobial activity of PBE was produced by its bacteriostatic agents: ethylparaben $(0.5 \mathrm{mg} / \mathrm{mL}, 0.05 \%)$ and alcohol[36, 37]. Other studies have found that ethylparaben, as an antimicrobial preservative, could inhibit different types of bacteria and fungi $[38,39]$. Therefore, those data support that PBE may exert its effect through the two bacteriostatis agents, ethylparaben and alcohol, to inhibit $S$. aureus growth, and thus inhibiting bacterial conjunctivitis. Concerning the side effects of mulberry leaves, acute toxicity and 30-day subacute toxicity tests of MLE have proved that no pathological changes were observed in experimental animals even at high doses [30]. As MLE eye drops did not contain any preservatives, they are likely safer than many conventional drugs for conjunctivitis and are less prone to drug resistance as a short-term treatment for acute conjunctivitis.

The NLRP3 inflammasome, a multimeric protein complex, is the most fully characterized of the inflammasomes and consists of an upstream sensor protein (NLRP3), an adapter protein (apoptosisassociated speck-like protein containing a caspase recruitment domain, ASC), and a proinflammatory caspase (caspase-1) (Schroder et al., 2010). Furthermore, our results indicated that the NLRP3 inflammasome pathway was significantly activated in the infected-conjunctiva. An earlier study demonstrated an increase in the expression of $N L R P 3$ in the conjunctiva of children with non-chlamydial bacterial infection [40], which supports our findings. Treatment with MLE and PBE markedly reduced the augmented expression of the NLRP3 inflammasome compared to the conjunctivitis model group. In contrast, a previous study found that combined mulberry leaf and fruit extract supplementation for 12 weeks could accelerate wound healing through activation of the NLRP3 inflammasome in high-fat dietinduced obese mice [41]. It has been reported that mulberry fruits, which are rich in p-coumaric acid, rutin, and quercetin, have strong immunomodulatory effects on splenocytes by decreasing two types of CD $4+$ T-helper lymphocytes (Th1/Th2) and pro-/anti-inflammatory cytokine secretion ratios [42]. Therefore, we postulated that the regulatory effect of mulberry leaves on the NLRP3 inflammasome might also be attributed to their immunomodulatory potential.

Several related studies have revealed that conjunctivitis can cause systemic inflammatory responses involving the induction of numerous inflammatory cytokines, including IL-1 $\beta$, TNF-a, and IL- 6 , which are also involved in an autocrine loop of inflammatory mediators [27, 43-45]. The production of cytokines by tissue macrophages and other immune cells is frequently induced by pathogen-associated molecular patterns, which act via NOD-like receptors (NLRs) [46]. When the NLRP3 inflammasome is activated, the mature and active forms of cytokines, such as IL-1 $\beta$ and IL-18, are secreted and induce inflammation [47]. Inflammation is induced by inflammasome activation, and acute inflammation, which is considered to be 
an attempt of the tissue to cope with the stressor and to restore a stress-free state, might be beneficial for the tissue and the whole organism [48]. However, the activation of inflammation-associated pathways is harmful at the cell-level, as it causes cell death. The release of IL-1 $\beta$, IL-18, and other inflammatory factors as a result of inflammasome activation can induce programmed pro-inflammatory cell death (pyroptosis) [49]. Consequently, attenuation of pathogen-induced inflammasome activation can protect the conjunctiva against inflammation and cell death. As expected, treatment with $\mathrm{MLE}\left(5 \mathrm{~mL} / \mathrm{kg} \cdot \mathrm{d}^{-1}\right.$ and $10 \mathrm{~mL} / \mathrm{kg} \cdot \mathrm{d}^{-1}$ ) and PBE significantly reduced the transcription of the NLRP3 inflammasome (Caspase-1, ASC, and NLRP3) and pro-inflammatory cytokines (IL-18, IL-6, and IL-1 $\beta$ ).

The Kelch-like ECH-associated protein 1 (Keap1)-Nrf2 system is a key player in cytoprotection and is activated under stress conditions. Several reports have proposed a protecting role of Nrf2 in infection and an inverse correlation between infection and a decline in Nrf2 activity [50-52]. Additionally, Nrf2 directly prevents the transcription of genes encoding the pro-inflammatory cytokines IL-6, prolL-1 $\beta$, and prolL-1a $[53,54]$. Our results showed that MLE markedly activated the Keap1-Nrf2 system in a non-concentration dependent manner, while PBE only significantly elevated Nrf2 expression but did not affect Keap1 expression. Therefore, the protective effect of MLE and PBE against bacterial conjunctivitis maybe associated with activated the Nrf2 pathway.

Moreover, there are several reports demonstrating the anti-inflammatory effects of Nrf2-activating compounds in different disease models associated with NLRP3 inflammasome inhibition [11]. Our study also showed that the coordinated regulation of Nrf2 and NLRP3 may play a crucial role in the protective effect of MLE and PBE in S. aureus-induced conjunctivitis in rabbits. According to a recent review [34] and to the best of our knowledge, this is the first demonstration of promising anti-inflammatory and antibacterial activity of MLE in bacterial conjunctivitis and that it is associated with Nrf2 activation and NLRP3 inflammasome inhibition; however, further analysis of the active ingredients potentially involved in this process and its molecular mechanisms is needed.

\section{Conclusions}

In summary, our data demonstrated that MLE is effective for the treatment of $S$. aureus-induced conjunctivitis in rabbits and its effect may be largely owing to its inhibition of the NLRP3 inflammasome and activation of the Nrf2 system to regulate pro-inflammatory signaling. Therefore, mulberry leaves might be a new and attractive phytomedicine for infectious conjunctivitis.

\section{Abbreviations}

ASC, apoptosis-associated speck-like protein containing a caspase recruitment domain, CFU, colonyforming units, HMLE, high-dose mulberry leaf extract, Keap1, Kelch-like ECH-associated protein 1, LMLE, low-dose mulberry leaf extract, MLE, mulberry leaf extract, NLRP3, NOD-like receptor leucine-rich pyrin domain-containing protein 3, Nrf2, nuclear factor erythroid 2-related factor 2, PED, pearl eye drops, qPCR, quantitative real-time reverse transcription PCR

Page $10 / 20$ 


\section{Declarations}

\section{Acknowledgments}

This study was supported by The Project Natural Science Foundation of China (No. 81760697), The Science and Technology Foundation of Guizhou Province of China (QKHJC [2017]1220), and The open topic of Key Laboratory of Basic Pharmacology of Ministry of Education and Joint International Research Laboratory of Ethnomedicine of Ministry of Education (Zunyi Medical University), Education Department of Guizhou Province of China (QJHKY[2020]253).

\section{Authors' contributions}

YC, LLL and ENL are involved in the bench work, data acquisition and analysis. YC, LLL, YYZ and XL perform experiments and data analysis. WNL is involved in the design and organization of the study, interpretation of the results, and the preparation of the manuscript. All authors have read and approved the final manuscript.

\section{Funding sources}

This research did not receive any specific grant from funding agencies in the public, commercial, or notfor-profit sectors.

\section{Ethics approval and consent to participate}

All experiments procedures and animal welfare were carried out in accordance with the "Guidelines for the Management and Use of Laboratory Animals"(Ministry of Science and Technology of China, 2006) and approved by the Zhuhai Campus Zunyi Medical University Institutional Animal Ethics and Utilization Committee.

\section{Declarations of interest:}

None

\section{Consent for publication}

Not applicable.

\section{Competing interests}

Conflict of interest On behalf of all authors, the corresponding author states. That there is no conflict of interest.

\section{References}


1. Liang QF, Du XH, Su GY, Zhang Y, Wang ZQ, Cao K: Genetic typing and susceptibility testing of strains from Staphylococcus aureus keratitis or conjunctivitis patients. Zhonghua Yan Ke Za Zhi 2018, 54:767-74.

2. O'Callaghan RJ: The pathogenesis of Staphylococcus aureus eye Infections. Pathogens 2018, 7.

3. Astley R, Miller FC, Mursalin MH, Coburn PS, Callegan MC: An eye on Staphylococcus aureus toxins: roles in ocular damage and inflammation. Toxins (Basel) 2019, 11:356.

4. Cavuoto K, Zutshi D, Karp CL, Miller D, Feuer W: Update on bacterial conjunctivitis in South Florida. Ophthalmology 2008, 115:51-6.

5. Haas W, Pillar CM, Torres M, Morris TW, Sahm DF: Monitoring antibiotic resistance in ocular microorganisms: results from the Antibiotic Resistance Monitoring in Ocular micRorganisms (ARMOR) 2009 surveillance study. American Journal of Ophthalmology 2011, 152:567-74.e.3.

6. Hovding G: Acute bacterial conjunctivitis. Acta Ophthalmol 2008, 86:5-17.

7. Wada T, Kida T, Inoue T, Tokushige H, Naka H, Sakaki H: Immunomodulatory effect of gatifloxacin on mouse peritoneal macrophages in vitro and in models of endotoxin-induced rat conjunctivitis and rabbit bacterial keratitis. Ophthalmic Research 2008, 40:54-60.

8. Dogru M, Kojima T, Simsek C, Tsubota K: Potential Role of Oxidative Stress in Ocular Surface Inflammation and Dry Eye Disease. Investigative Ophthalmology \& Visual Science 2018, 59:DES163DES8.

9. Federico A, Morgillo F, Tuccillo C, Ciardiello F, Loguercio C: Chronic inflammation and oxidative stress in human carcinogenesis. International Journal of Cancer 2007, 121:2381-6.

10. Wakamatsu TH, Dogru M, Ayako I, Takano Y, Matsumoto Y, Ibrahim OMA, Okada N, Satake Y, Fukagawa K, Shimazaki J, et al: Evaluation of lipid oxidative stress status and inflammation in atopic ocular surface disease. Molecular Vision 2010, 16:2465-75.

11. Hennig P, Garstkiewicz M, Grossi S, Di Filippo M, French LE, Beer HD: The crosstalk between Nrf2 and inflammasomes. International Journal of Molecular Sciences 2018, 19:562.

12. Ahmed SM, Luo L, Namani A, Wang XJ, Tang X: Nrf2 signaling pathway: pivotal roles in inflammation. Biochimica et Biophysica Acta (BBA) - Molecular Basis of Disease 2017, 1863:585-97.

13. Jhang JJ, Yen GC: The role of Nrf2 in NLRP3 inflammasome activation. Cellular and Molecular Immunology 2017, 14:1011-2.

14. Committee SP: Pharmacopoeia of the People's Republic of China. China Medical Science and Technology Press 2015, 1:297-310.

15. El-Sayyad HIH, El-Sherbiny MA, Sobh MA, Abou-El-Naga AM, Ibrahim MAN, Mousa SA: Protective effects of Morus alba leaves extract on ocular functions of pups from diabetic and hypercholesterolemic mother rats. International Journal of Biological Sciences 2011, 7:715-28.

16. Assiri AMA, El-Beeh ME, Amin AH, Ramadan MF: Ameliorative impact of Morus alba leaves' aqueous extract against embryonic ophthalmic tissue malformation in streptozotocin-induced diabetic rats. Biomedicine \& Pharmacotherapy 2017, 95:1072-81. 
17. Mahmoud AM, El-Twab SMA, Abdel-Reheim ES: Consumption of polyphenol-rich Morus alba leaves extract attenuates early diabetic retinopathy: the underlying mechanism. European Journal of Nutrition 2017, 56:1671-84.

18. Chiu HF, Hsiao SC, Lu YY, Han YC, Shen YC, Venkatakrishnan K, Wang CK: Efficacy of protein rich pearl powder on antioxidant status in a randomized placebo-controlled trial. Journal of Food and Drug Analysis 2018, 26:309-17.

19. Yang HL, Korivi M, Lin MK, Chang HC, Wu CR, Lee MS, Chen WT, Hseu YC: Antihemolytic and antioxidant properties of pearl powder against 2,2'-azobis (2-amidinopropane) dihydrochlorideinduced hemolysis and oxidative damage to erythrocyte membrane lipids and proteins. Journal of Food and Drug Analysis 2017, 25:898-907.

20. Zhang JX, Li SR, Yao S, Bi QR, Hou JJ, Cai LY, Han SM, Wu WY, Guo DA: Anticonvulsant and sedativehypnotic activity screening of pearl and nacre (mother of pearl). Journal of Ethnopharmacology 2016, 181:229-35.

21. Wang S, Zhang D, Hu J, Jia Q, Xu W, Su D, Song H, Xu Z, Cui J, Zhou M, et al: A clinical and mechanistic study of topical borneol-induced analgesia. EMBO Molecular Medicine 2017, 9:802-15.

22. Zhou HH, Zhang L, Zhou QG, Fang Y, Ge WH: (+)-Borneol attenuates oxaliplatin-induced neuropathic hyperalgesia in mice. Neuroreport 2016, 27:160-5.

23. Sloop GD, Moreau JM, Conerly LL, Dajcs JJ, O'Callaghan RJ: Acute inflammation of the eyelid and cornea in Staphylococcus keratitis in the rabbit. Investigative Ophthalmology \& Visual Science 1999, 10:385-91.

24. Tang A, Balzli CL, Caballero AR, McCormick CC, Taylor SD, O'Callaghan RJ: Staphylococcus aureus infection of the rabbit cornea following topical administration. Current Eye Research 2012, 37:107583.

25. Zhang Z, Abdel-Razek O, Hawgood S, Wang G: Protective role of surfactant protein D in ocular Staphylococcus aureus linfection. PLoS One 2015, 10:e0138597.

26. Magone MT, Chan C-C, Rizzo LV, Kozhich AT, Whitcup SM: A novel murine model of allergic conjunctivitis. Clinical Immunology and Immunopathology 1998, 87: 75-84.

27. Tanaka J, Tanaka H, Mizuki N, Nomura E, Ito N, Nomura N, Yamane M, Hida T, Goshima Y, Hatano H, Nakagawa $\mathrm{H}$ : Semaphorin $3 \mathrm{~A}$ controls allergic and inflammatory responses in experimental allergic conjunctivitis. International Journal of Ophthalmology 2015, 8:1-10.

28. Peng $\mathrm{CH}$, Lin HT, Chung DJ, Huang CN, Wang CJ: Mulberry Leaf Extracts prevent obesity-induced NAFLD with regulating adipocytokines, inflammation and oxidative stress. Journal of Food and Drug Analysis 2018, 26:778-87.

29. Li Y, Zhang X, Liang C, Hu J, Yu Z: Safety evaluation of mulberry leaf extract: Acute, subacute toxicity and genotoxicity studies. Regulatory Toxicology and Pharmacology 2018, 95:220-6.

30. Liu Y, Li X, Xie C, Luo X, Bao Y, Wu B, Hu Y, Zhong Z, Liu C, Li M: Prevention effects and possible molecular mechanism of mulberry leaf extract and its formulation on rats with insulin-insensitivity. PLoS One 2016, 11:e0152728. 
31. He X, Fang J, Ruan Y, Wang X, Sun Y, Wu N, Zhao Z, Chang Y, Ning N, Guo H, Huang L: Structures, bioactivities and future prospective of polysaccharides from Morus alba (white mulberry): A review. Food Chem 2018, 245:899-910.

32. Kwon DH, Jeong JW, Choi EO, Lee HW, Lee KW, Kim KY, Kim SG, Hong SH, Kim GY, Park C, et al: Inhibitory effects on the production of inflammatory mediators and reactive oxygen species by Mori folium in lipopolysaccharide-stimulated macrophages and zebrafish. Anais Da Academia Brasileira de Ciências 2017, 89:661-74.

33. Gryn-Rynko A, Bazylak G, Olszewska-Slonina D: New potential phytotherapeutics obtained from white mulberry (Morus alba L.) leaves. Biomedicine \& Pharmacotherapy 2016, 84:628-36.

34. Rodrigues EL, Marcelino G, Silva GT, Figueiredo PS, Garcez WS, Corsino J, Guimaraes RCA, Freitas $\mathrm{KC}$ : Nutraceutical and medicinal potential of the Morus species in metabolic dysfunctions. International Journal of Molecular Sciences 2019, 20:301.

35. Azari AA, Barney NP: Conjunctivitis: a systematic review of diagnosis and treatment. JAMA 2013, 310:1721-9.

36. Tang Z, Chen B: The preliminary study of medicine effect of pearl bright eyedrop. Strait Phaemaceutical Jornal 2000, 12:32-4.

37. Shen J, Yi X, Xu X, Wang Y, Lu X: Study on Antimicrobial Effectiveness of Zhenzhu Mingmu Eye Drops. China Pharmacist 2015, 18:894-6.

38. Jianmei C, Bo L, Zheng C, Huai S, Guohong L, Cibin G: Identification of ethylparaben as the antimicrobial substance produced by Brevibacillus brevis FJAT-0809-GLX. Microbiol Res 2015, 172:48-56.

39. Sun L, Yu T, Guo J, Zhang Z, Hu Y, Xiao X, Sun Y, Xiao H, Li J, Zhu D, et al: The estrogenicity of methylparaben and ethylparaben at doses close to the acceptable daily intake in immature SpragueDawley rats. Sci Rep 2016, 6:25173.

40. Burton MJ, Ramadhani A, Weiss HA, Hu V, Massae P, Burr SE, Shangali W, Holland MJ, Mabey DC, Bailey RL: Active trachoma is associated with increased conjunctival expression of IL17A and profibrotic cytokines. Infection and Immunity 2011, 79:4977-83.

41. Eo H, Lim Y: Combined mulberry leaf and fruit extract improved early stage of cutaneous wound healing in high-fat diet-induced obese mice. Journal of Medicinal Food 2016, 19:161-9.

42. Liu CJ, Lin JY: Anti-inflammatory effects of phenolic extracts from strawberry and mulberry fruits on cytokine secretion profiles using mouse primary splenocytes and peritoneal macrophages. International Immunopharmacology 2013, 16:165-70.

43. Chen $Y$, Hong $X$ : Effects of carvedilol reduce conjunctivitis through changes in inflammation, NGF and VEGF levels in a rat model. Experimental and Therapeutic Medicine 2016, 11:1987-92.

44. Wei CC, Kung YJ, Chen CS, Chang CY, Lin CJ, Tien PT, Chang HY, Chen HJ, Huang YS, Lin HJ, Wan L: Allergic conjunctivitis-induced retinal inflammation promotes myopia progression. EBioMedicine 2018, 28:274-86. 
45. Enriquez-de-Salamanca A, Calder V, Gao J, Galatowicz G, Garcia-Vazquez C, Fernandez I, Stern ME, Diebold Y, Calonge M: Cytokine responses by conjunctival epithelial cells: an in vitro model of ocular inflammation. Cytokine 2008, 44:160-7.

46. Taniguchi K, Karin M: NF-kappaB, inflammation, immunity and cancer: coming of age. Nature Reviews Immunology 2018, 18:309-24.

47. Strowig T, Henao-Mejia J, Elinav E, Flavell R: Inflammasomes in health and disease. Nature 2012, 481:278-86.

48. Medzhitov R: Origin and physiological roles of inflammation. Nature 2008, 454:428-35.

49. Fink SL, Cookson BT: Apoptosis, pyroptosis, and necrosis: mechanistic description of dead and dying eukaryotic cells. Infection and Immunity 2005, 73:1907-16.

50. Deramaudt TB, Dill C, Bonay M: Regulation of oxidative stress by Nrf2 in the pathophysiology of infectious diseases. Médecine et Maladies Infectieuses 2013, 43:100-7.

51. Medvedev R, Ploen D, Spengler C, Elgner F, Ren H, Bunten S, Hildt E: HCV-induced oxidative stress by inhibition of Nrf2 triggers autophagy and favors release of viral particles. Free Radical Biology and Medicine 2017, 110:300-15.

52. Staitieh BS, Ding L, Neveu WA, Spearman P, Guidot DM, Fan X: HIV-1 decreases Nrf2/ARE activity and phagocytic function in alveolar macrophages. Journal of Leukocyte Biology 2017, 102:517-25.

53. Suzuki T, Yamamoto M: Stress-sensing mechanisms and the physiological roles of the Keap1-Nrf2 system during cellular stress. Journal of Biological Chemistry 2017, 292:16817-24.

54. Kobayashi EH, Suzuki T, Funayama R, Nagashima T, Hayashi M, Sekine H, Tanaka N, Moriguchi T, Motohashi H, Nakayama K, Yamamoto M: Nrf2 suppresses macrophage inflammatory response by blocking proinflammatory cytokine transcription. Nature Communications 2016, 7:11624.

\section{Tables}

Due to technical limitations, tables are only available as a download in the Supplemental Files section.

\section{Figures}




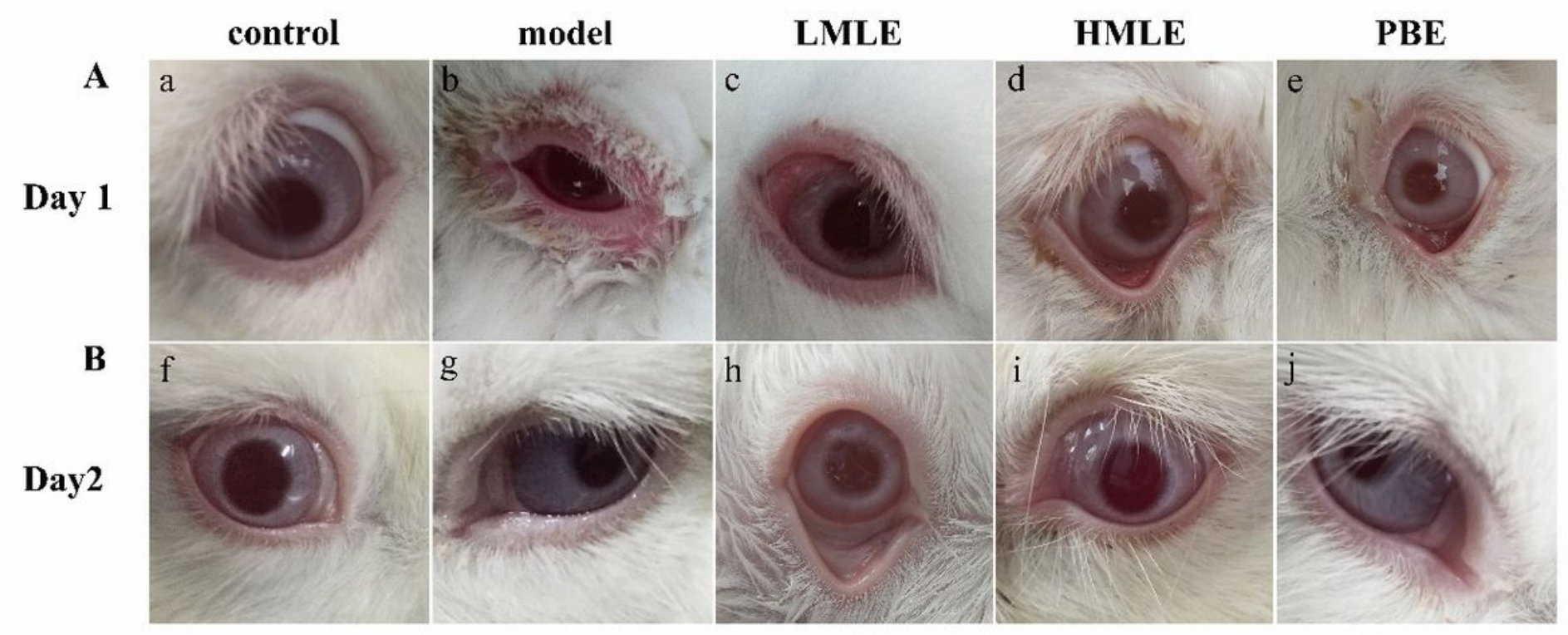

Figure 1

Clinical signs on Day 1 (A) and Day 5 (B). The eye was normal in control group (a, $f)$. In the model group (b) and three administration groups (c, d, e), the conjunctiva was infected with obvious congestion, exudation, edema and ectropion of eyelid to nearly closed after $\mathrm{S}$. aureus infected. The conjunctiva had a great improvement with a small amount of mucus on Day 5 in LMLE group (h). The conjunctiva nearly recovered on Day 5 in (i) HMLE group, and (j) PBE group.

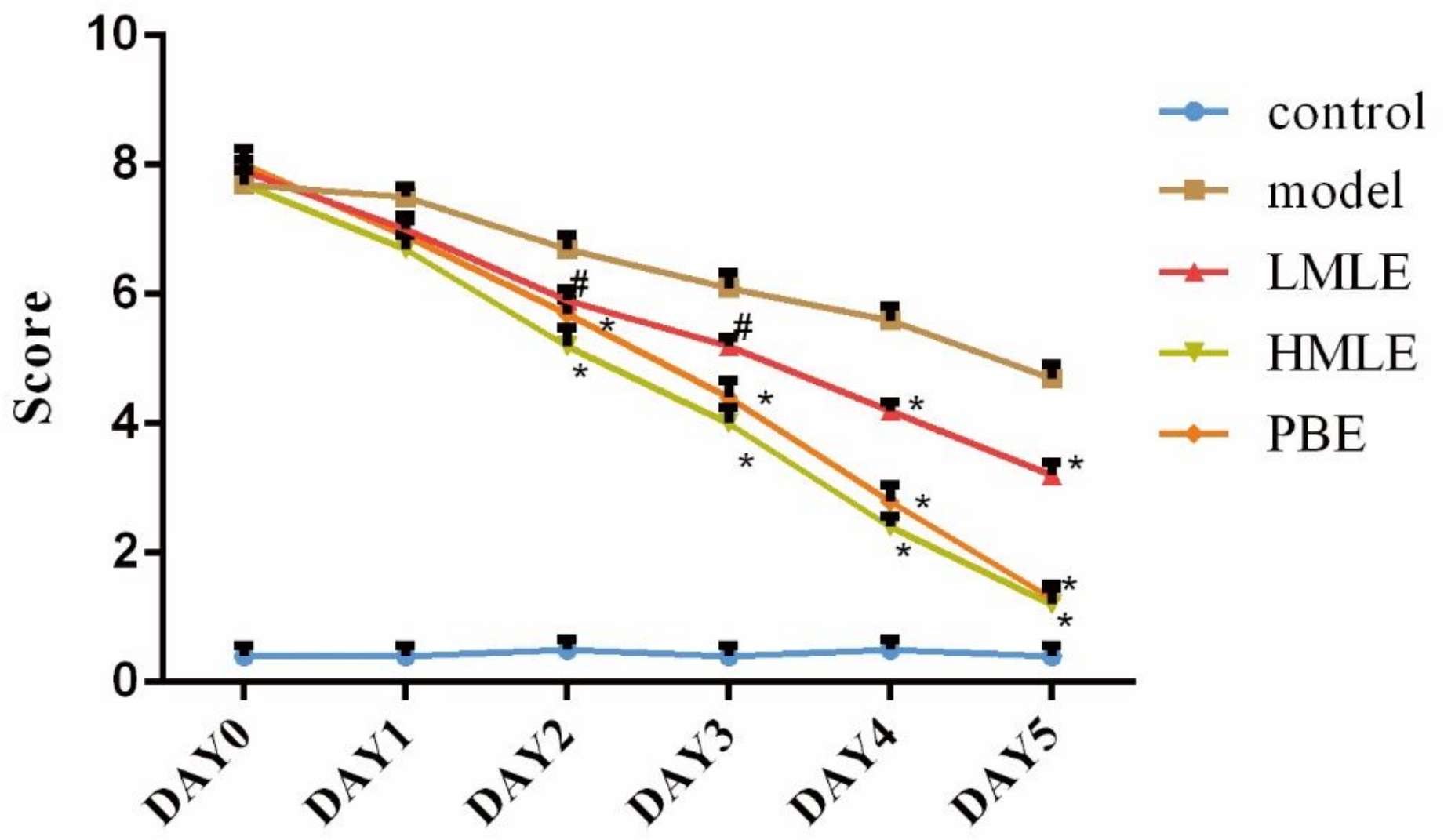


Figure 2

Clinical score of inflammation and anti-inflammatory effect of MLE in a rabbit conjunctivitis model induced by S. aureus. The effect of MLE was evaluated by total score from 0 to 9 , with $0 \rrbracket 2$ for cure, $2 \varangle 5$ for significant effect, $5 \llbracket 8$ for improvement, $8 \sim 9$ for ineffectiveness. Data are expressed as mean \pm SEM $(n=5)$ from at least ten independent experiments. \#P $\leq 0.05$ vs. model, ${ }^{*} \leq 0.01$ vs. model

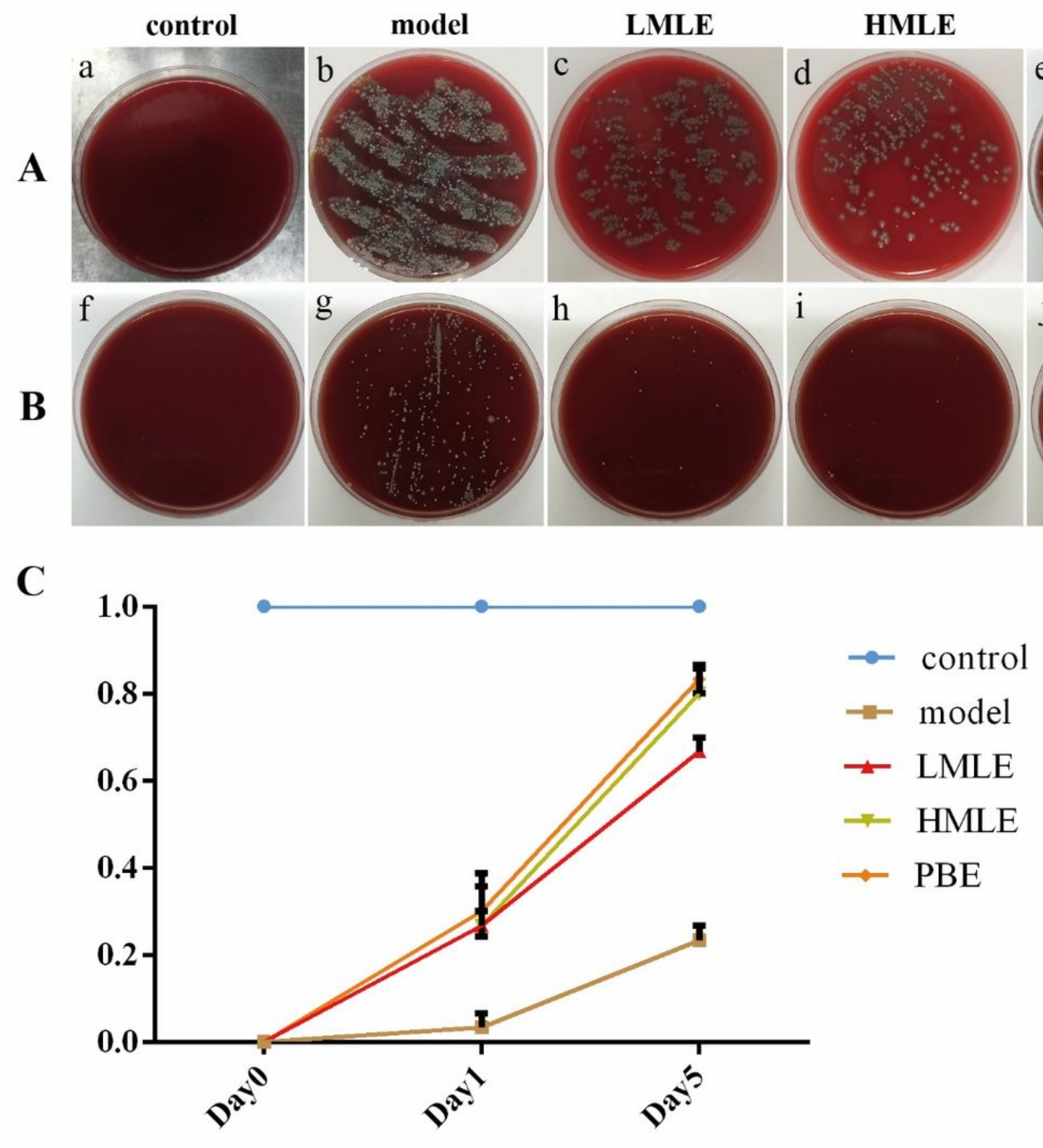

Figure 3

Compared the Colony of S. aureus on Day $1(\mathrm{~A})$ or Day $5(\mathrm{~B})$, and bacterium negative conversion rate of rabbits on Day 0, Day 1, and Day 5 (C). The model group (b) and three administration groups (c, d, e) on 
Day 0 produced lots of colonies and had typical morphology characteristics which was arranged as grape-like clusters, with a smooth surface, golden or colorless, opaque. In contrast, there were only a few colonies can be seen on LMLE group (h), while almost no colony were produced in HMLE group (i) and PBE group (j) on Day 5.

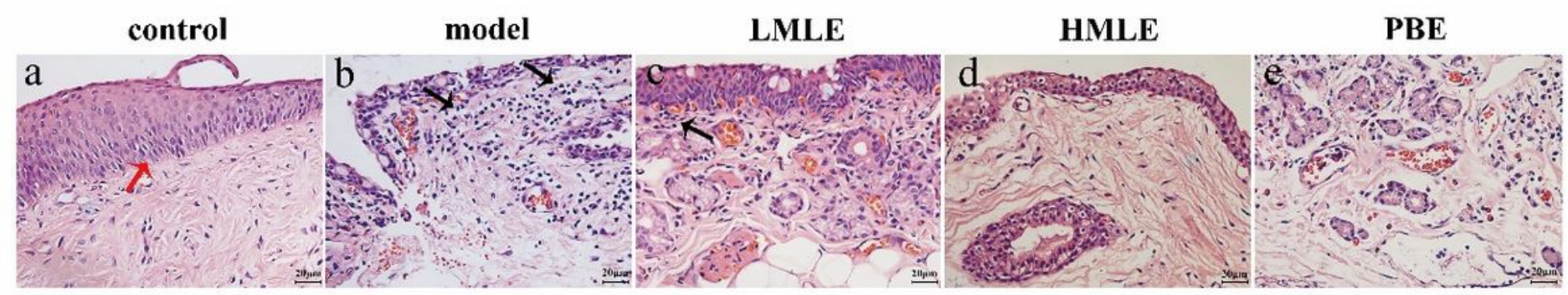

\section{Figure 4}

Representative histopathologic pictures of rabbit conjunctival tissues on Day 5. The control group (a) showed no diagnostic abnormalities, and the structure of stratified columnar epithelial tissue is clear (red arrow) on Day5. However, serious inflammatory occurred (b) with dense lymphocytes infiltrate (black arrow), telangiectasia, congestion and edema in submucosal connective tissue. LMLE group eyes (c) began to resolve, but still showed a few inflammatory cells infiltration and capillary congestion, while in HMLE group (d) and PBE group (e) showed effective clinical cure after treated for 5 days. (H\&E stain; original magnification $\times 400)$.
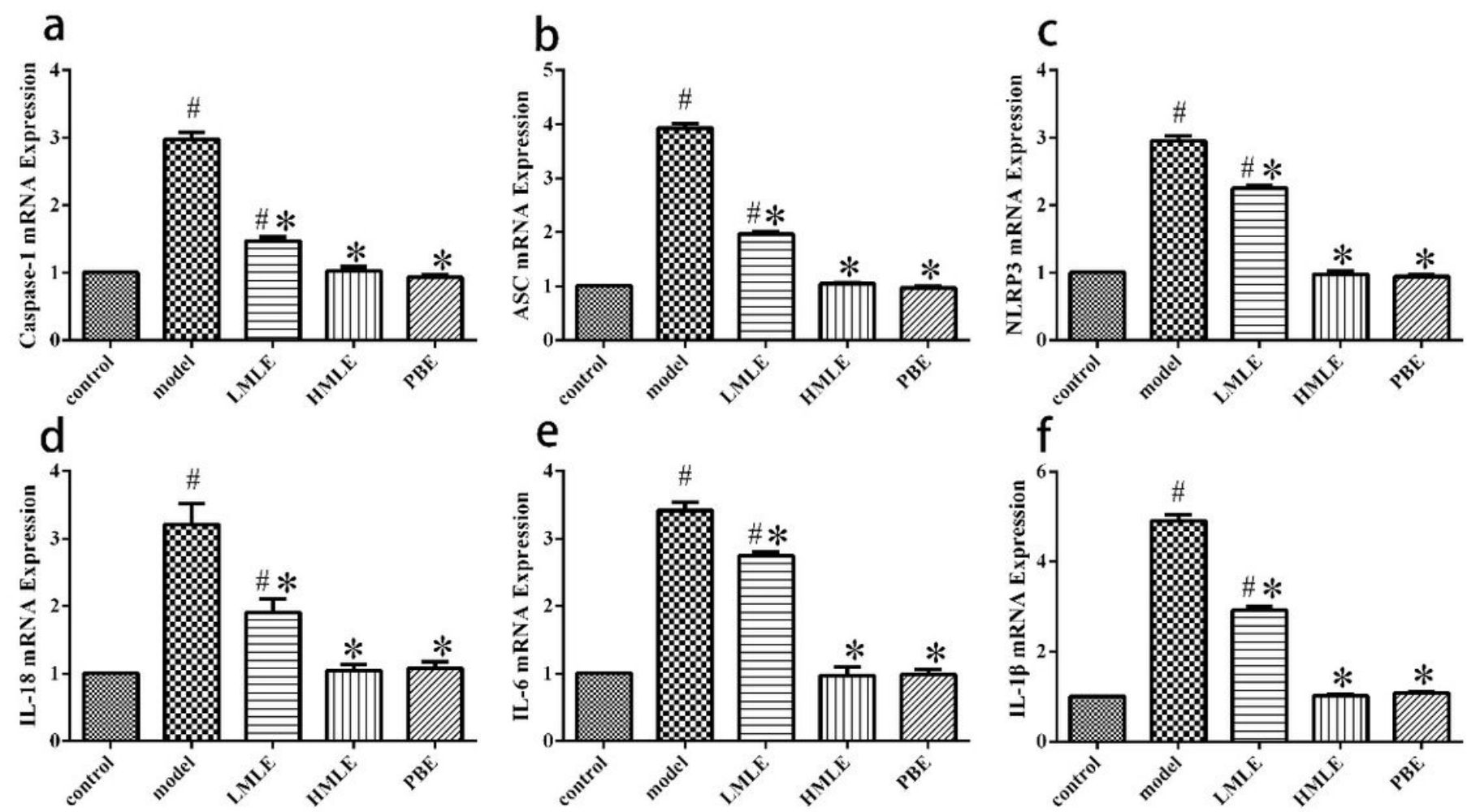

Figure 5 
Effects of MLE on the mRNA expression levels of NLRP3 inflammasome (Caspase-1(Fig. 5a), ASC (Fig. 5b), NLRP3 (Fig. 5c)) and its downstream pro-inflammatory cytokines (IL-18 (Fig. 5d), IL-6 (Fig. 5e) and IL-1 $\beta$ (Fig. 5f)) in a rabbit model of conjunctivitis on Day 5. All genes were normalized with GAPDH. Data represent as mean \pm SEM $(n=5)$ of three independent experiments. \#P $\leq 0.05$ vs. control, *P $\leq 0.05$ vs. model
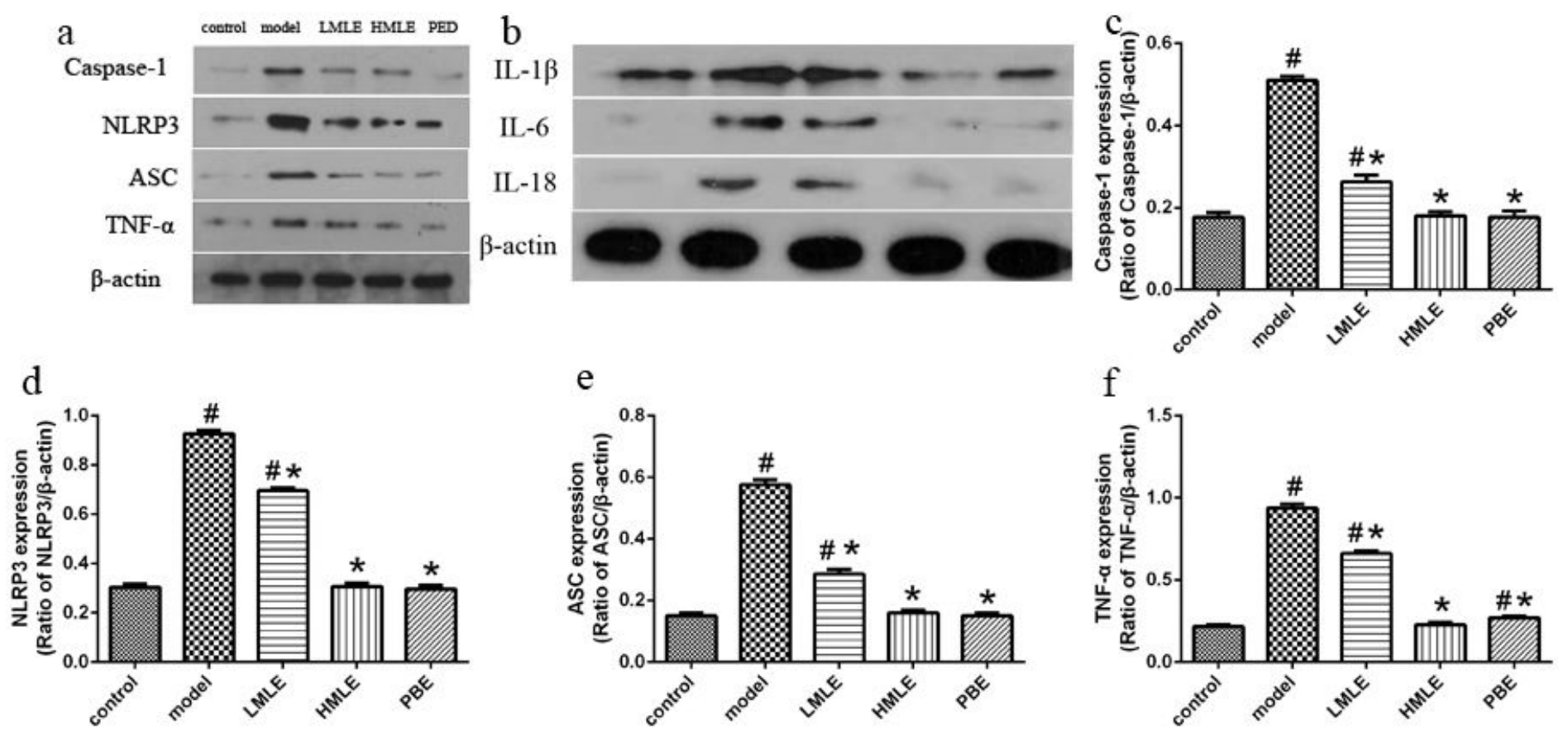

$\mathrm{f}$
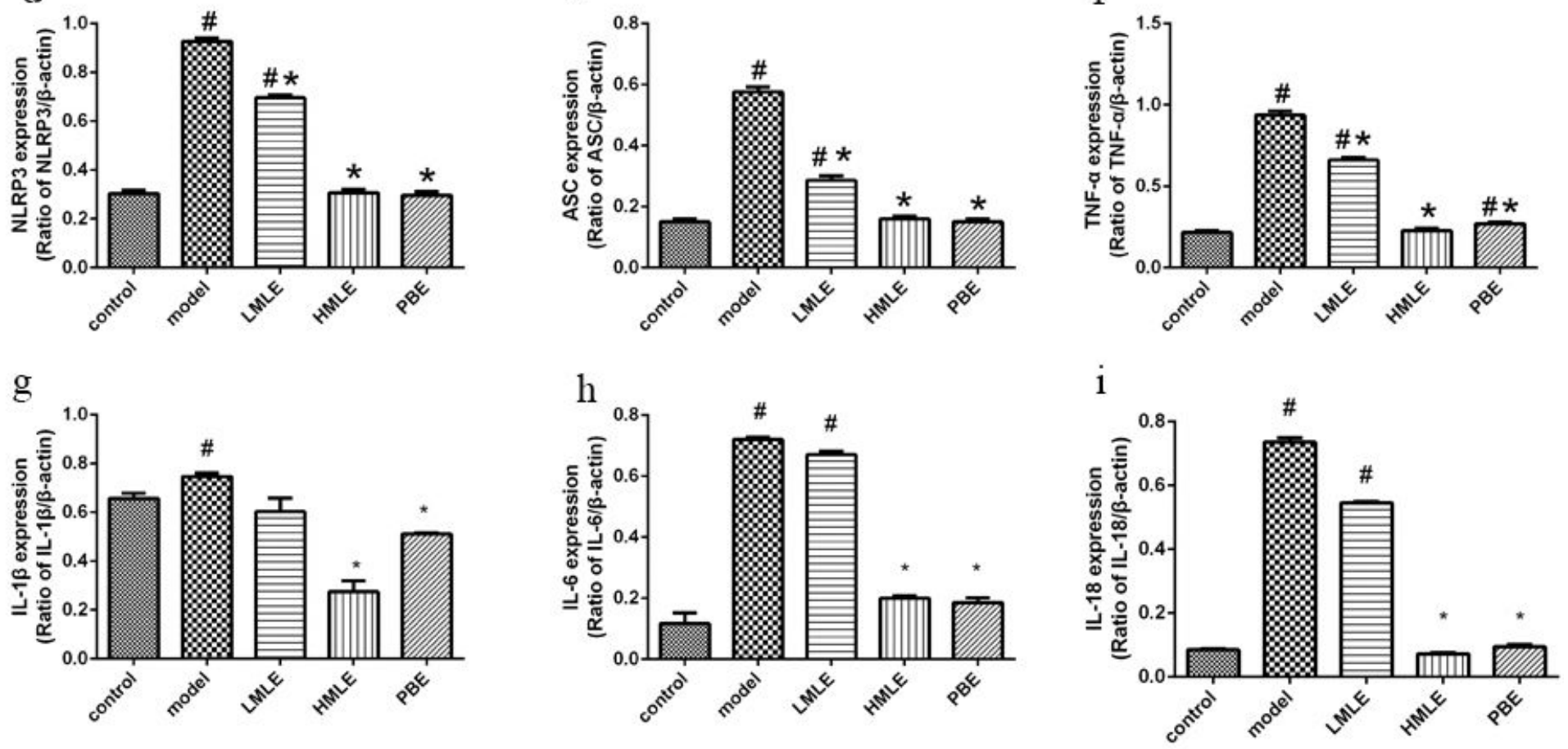

Figure 6

Effects of MLE on the protein levels of NLRP3 inflammasome and downstream pro-inflammatory factors in a rabbit model of conjunctivitis on Day 5. (a-b) Western blotting analysis of NLRP3 inflammasome (Caspase-1, NLRP3, ASC) and pro-inflammatory factors (IL-18, TNF-a, IL-6 and IL-1 3 ); (c-i) The densitonmetric scanning of Caspase-1, NLRP3, ASC, TNF- $a$, IL-1 $\beta$, IL- 6 and IL-18 after normalization with $\beta$-actin. Data represent as mean $\pm S E M$ of three independent experiments. $\# P \leq 0.05$ vs. control, ${ }^{*} P \leq 0.05$ vs. model 
a

control model LMLE HMLE PBE
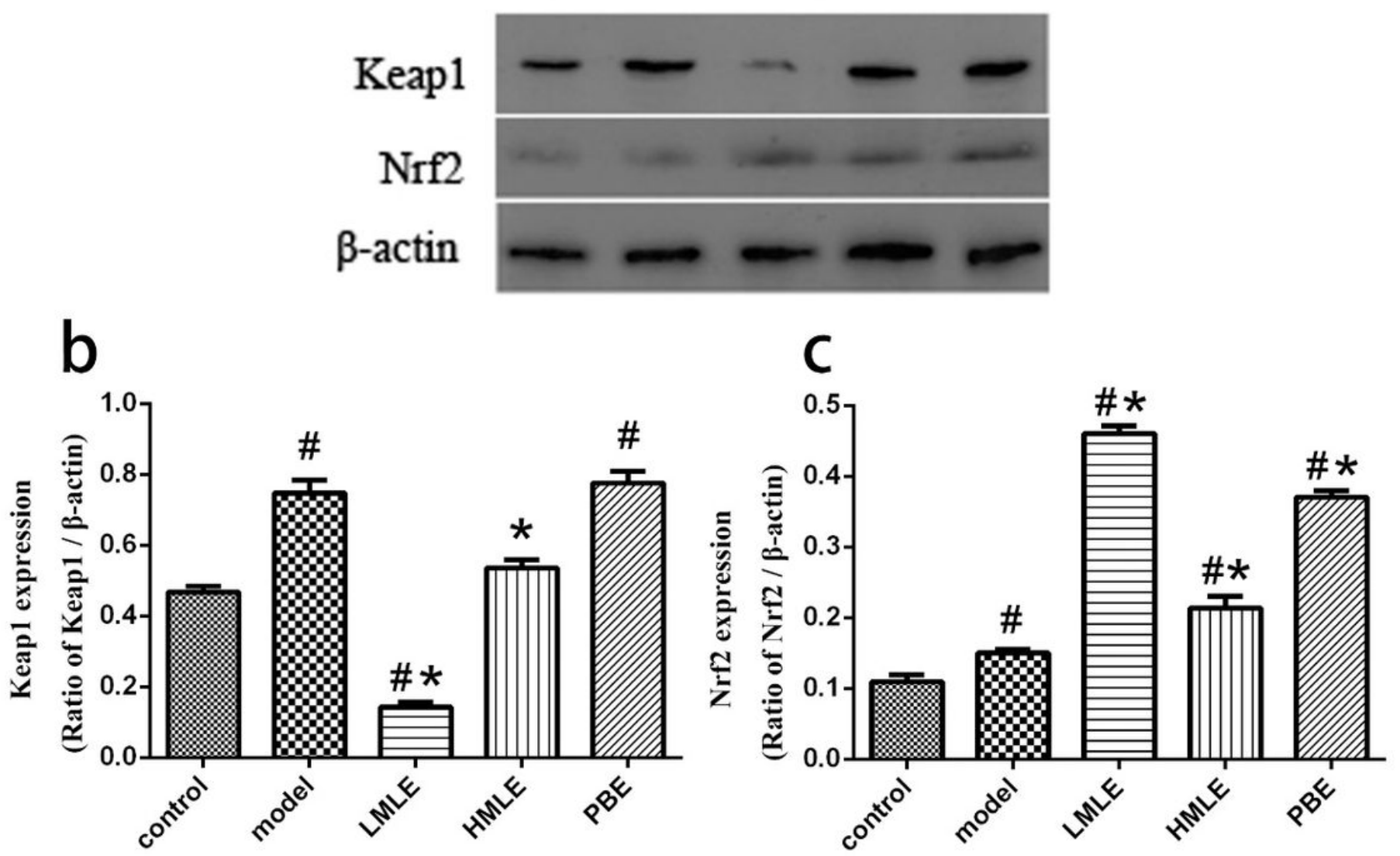

Figure 7

Effects of MLE on the protein levels of Keap1 and Nrf2 in a rabbit model of conjunctivitis on Day 5. a Western blotting analysis of Keap1 and Nrf2, $(b, c)$ The densitonmetric scanning of Keap1 and Nrf2 after normalization with $\beta$-actin. Data represent as mean \pm SEM of three independent experiments. $\# P \leq 0.05$ vs. control, ${ }^{*} \mathrm{P} \leq 0.05$ vs. model

\section{Supplementary Files}

This is a list of supplementary files associated with this preprint. Click to download.

- tables.docx 